Virginia Commonwealth University VCU Scholars Compass

1993

\title{
Phenomenological theory of cuprate superconductivity
}

Mario Rabinowitz

Electric Power Research Institute

T. McMullen

Virginia Commonwealth University

Follow this and additional works at: http://scholarscompass.vcu.edu/phys_pubs

Part of the Physics Commons

Rabinowitz, M., McMullen, T. Phenomenological theory of cuprate superconductivity. Applied Physics Letters, 63, 985 (1993). Copyright (C) 1993 AIP Publishing LLC.

\section{Downloaded from}

http://scholarscompass.vcu.edu/phys_pubs/46

This Article is brought to you for free and open access by the Dept. of Physics at VCU Scholars Compass. It has been accepted for inclusion in Physics Publications by an authorized administrator of VCU Scholars Compass. For more information, please contact libcompass@vcu.edu. 


\title{
Phenomenological theory of cuprate superconductivity
}

\author{
Mario Rabinowitz \\ Electric Power Research Institute, Palo Alto, California 94303 \\ T. McMullen \\ Department of Physics, Commonwealth University, Richmond, Virginia 23284
}

(Received 19 March 1993; accepted for publication 28 May 1993)

\begin{abstract}
Reasonably good agreement with the superconducting transition temperatures of the cuprate high- $T_{c}$ superconductors can be obtained on the basis of an approximate phenomenological theory. In this theory, two criteria are used to calculate the superconducting transition temperature. One is that the quantum wavelength is of the order of the electron-pair spacing. The other is that a fraction of the normal carriers exist as Cooper pairs at $T_{c}$. The resulting simple equation for $T_{c}$ contains only two parameters: the normal carrier density and effective mass. We calculate specific transition temperatures for 12 cuprate superconductors.
\end{abstract}

As the temperature of an ideal Bose gas is lowered, the particles undergo a Bose-Einstein (BE) condensation when the thermal wavelength

$$
\lambda_{T}=h /\left(2 \pi m_{B} k_{\beta} T_{c}\right)^{1 / 2}
$$

is comparable to the interparticle spacing. ${ }^{1,2}$ Here, $h$ is Planck's constant, $k_{\beta}$ is the Boltzmann constant, $m_{B}$ is the boson mass, and $T_{c}$ is the transition temperature. $\lambda_{T}$ is called the thermal wavelength, because $\lambda_{T} \approx \lambda$, the de Broglie wavelength.

For a three-dimensional ideal Bose gas, the BE condensation temperature ${ }^{1,2}$ is

$$
T_{c}^{\mathrm{BE}}=\frac{h^{2} n^{2 / 3}}{2 \pi m_{B} k_{\beta}\left[\zeta\left(\frac{3}{2}\right)\right]^{2 / 3}}=\frac{h^{2} n^{2 / 3}}{11.92 m_{B} k_{\beta}},
$$

where $n$ is the three-dimensional number density, $\zeta(x)$ is the Riemann zeta function of $x$, and $\zeta(3 / 2)=2.612 \ldots$. There are two shortcomings related to the ordinary BE condensation. One is that $T_{c}^{\mathrm{BE}}$ is orders of magnitude higher than the experimental $T_{c}$ of three-dimensional superconductors like the metallics if a sizable fraction of the normal carriers are bosons. The other is that the $\mathrm{BE}$ gas does not condense in two dimensions so that unmodified $\mathrm{BE}$ statistics are not a sufficient condition for BE condensation. More general considerations argue against a onedimensional condensation. However, with an energy gap (binding energy between particles), a BE type condensation in lower dimensions is described by Blatt, ${ }^{3}$ although this appears to be contrary to Hohenberg's theorem. ${ }^{4}$

Two conditions are met by known superconductors. One is the existence of bosons. The other is a condensation in momentum space. This led to a consideration that there may be two temperatures: $T_{p}$, the pairing temperature to form bosons out of fermion pairs; and the condensation temperature $T_{C}$. If $T_{p}<T_{C}$, then $T_{p}$ is the limiting temperature for superconductivity which we call the transition temperature $T_{c}$. However, it is difficult to calculate $T_{p}$ because this requires knowledge of the pairing interaction and its strength, although this is the usual strategy. Three possibilities were considered first by Rabinowitz ${ }^{5-9}$ in 1987:

(1) That $T_{C}$ might be less than or equal to $T_{p}$, in which case $T_{\mathrm{c}}=T_{C}$.
(2) That a fraction of fermions $\sim k_{\beta} T_{c} / E_{F}$ are incipient Cooper pairs at $T_{c}$.

(3) Reduced dimensionality effects properly enter into $\lambda$ via the equipartition principle.

His paradigm led to good agreement with experiment for broad classes of superconducting materials from 1 to $10^{9}$ K. ${ }^{5-9}$ More recently this has been extended to include $10^{-3}$ $\mathrm{K}$ for the superfluid state of ${ }^{3} \mathrm{He}$ for a range of 12 orders of magnitude. ${ }^{10,11}$

Even if $T_{C}>T_{p}$, the $T_{C}$ is found to be a much better upper limit to $T_{c}$ than $T_{c}^{\mathrm{BE}}$. We are not aware of any $T_{c}$ calculations for specific cuprates, nor of any other attempts to calculate a reduced condensation temperature which does not include interactions. Here, we present a more detailed test of this approach by comparing its results with the observed $T_{c}$ 's of specific cuprates. Harshman and Mills $^{12}$ have compiled a comprehensive table of the relevant parameters, extracted from experimental data, which makes this possible. BE condensation approaches to superconductivity were first presented by Schafroth, Butler, and Blatt. ${ }^{13,14}$ These approaches differ from ours, and appear not to have been successful in predicting $T_{c}$ 's. Standard approaches have been unsuccessful with the cuprates.

The point of view adopted here is that as the temperature is decreased in a gas similar to a Bose-Einstein (BE) gas, particles should start a $\mathrm{BE}$ type condensation into the superconducting state when the de Broglie wavelength is of the order of the interparticle spacing for the fraction of carriers that have become Cooper pairs. ${ }^{5-11}$ Thus we can avoid the difficult calculations encountered when the nature of the interaction is known, and which are impossible to do when the pairing interaction is not known.

We assume that a BE type condensation in three dimensions of the free Bose gas involves electron pairs within a shell of energy $\sim k_{\beta} T_{c}$ around the Fermi energy, $E_{F}$. The number of electrons involved is the number of $k$ values within a shell $\Delta k$ of energy width $\sim k_{B} T_{c}$ within the Fermi surface, so that $\Delta k$ is given by

$$
k_{\beta} T_{c} \approx \frac{\hbar^{2} k_{F}^{2}}{2 m}-\frac{\hbar^{2}\left(k_{F}-\Delta k\right)^{2}}{2 m},
$$


TABLE I. Comparison of experimental and theoretical cuprate transition temperatures.

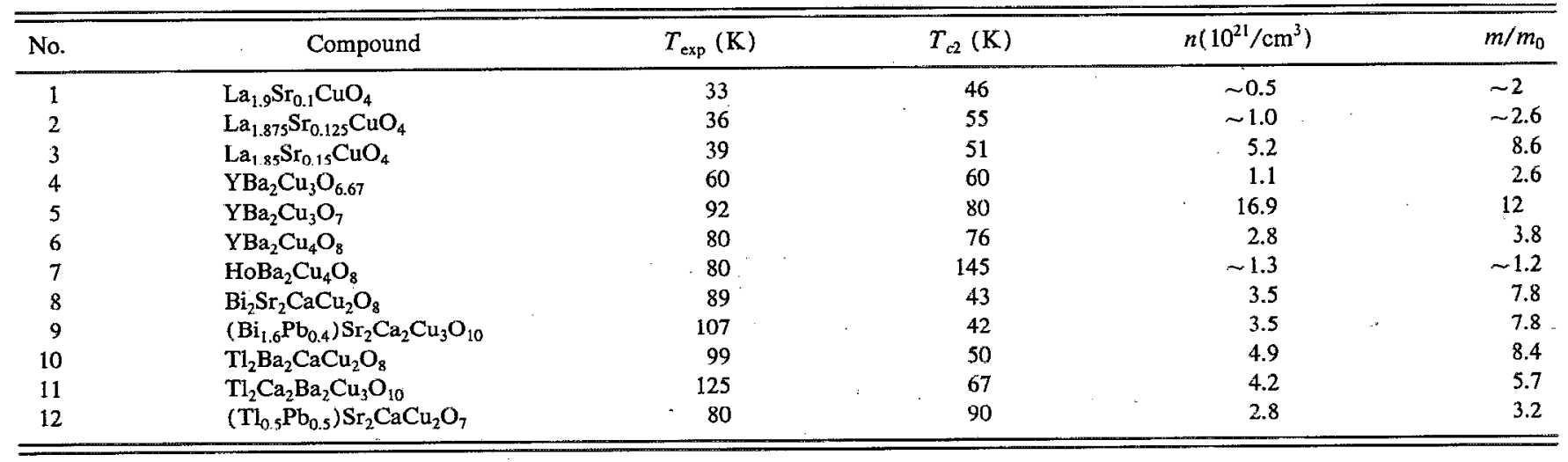

where $k$ is the wave vector and $m$ is the effective mass of the electron. Thus

$$
\frac{\Delta k}{k_{F}}=\frac{k_{\beta} T_{c}}{2\left[\left(\hbar^{2} / 2 m\right) k_{F}^{2}\right]}=\frac{k_{\beta} T_{c}}{2 E_{F}} .
$$

The number density of Cooper pairs for a roughly spherical Fermi surface is then.

$$
n_{B}=\frac{1}{2} \frac{\Delta k\left(4 \pi k_{F}^{2}\right)}{\frac{4}{3} \pi k_{F}^{3}} n=\frac{3 \Delta k}{2 k_{F}} n .
$$

Combining Eqs. (4) and (5), we have

$$
n_{B}=\frac{3 k_{\beta} T_{c 3}}{4 E_{F}} n,
$$

where we introduce $T_{c 3}$, a "three-dimensional" transition temperature that may apply to some less anisotropic materials, and which can be thought of here as providing the energy cutoff that selects the effective bosons. The procedure will be to use $T_{c 3}$ to determine the interboson spacing in order to calculate $T_{c 2}$, which will be our transition temperature estimate for the cuprates. The choice of "twodimensional" motion for these materials is based on their layered structure and highly anisotropic normal state properties.

Two electron pairs are encompassed by $(1 / 2) \lambda$ when

$$
\lambda \approx 2\left(n_{B}\right)^{-1 / 3} \text {. }
$$

For a pair of electrons of effective mass $2 m$, momentum $p$, and kinetic energy $(f / 2) k_{\beta} T$, the de Broglie wavelength is

$$
\lambda=\frac{h}{p}=\frac{h}{\left\{2(2 m)\left[(f / 2) k_{\beta} T_{c}\right]\right\}^{1 / 2}},
$$

where $f$ is the number of degrees of freedom per particle pair. For three-dimensional motion of the bosons we take $f=3$. For two-dimensional motion we will take $f=2$. Rabinowitz gives a more general discussion of $f$ in Ref. 7 . Combining Eqs. (6)-(8) we obtain

$$
T_{c 3}=0.218\left(\frac{\hbar^{2} n^{2 / 3}}{2 m k_{\beta}}\right) \text {. }
$$

To obtain the "two-dimensional" transition temperature, $\lambda$ as given by Eq. (7) is equated to $\lambda$ as given by Eq.
(8) with $f=2$, as we restrict the kinetic energy to two dimensions by the equipartition of energy principle. This yields the two-dimensional transition temperature which we will compare with experiment:

$$
T_{c 2}=0.328\left(\frac{\hbar^{2} n^{2 / 3}}{2 m k_{\beta}}\right) \text {. }
$$

Table I shows a comparison of the experimentally observed transition temperatures with $T_{c 2}$, together with the relevant input data as obtained from Harshman and Mills. ${ }^{12}$ In Eq. (10) we use the three-dimensional (3D) carrier density $n_{3 \mathrm{D}}$ for $n$, and the effective mass for $m$, where $m_{0}$ is the free-electron mass.

It is remarkable how well our calculated transition temperatures agree with the experimental values without explicitly introducing a pairing mechanism. Perhaps this should not be entirely surprising, as $T_{c}$ itself is a measure of the interaction strength. In this model, $T_{c}$ enters into the equations in two different ways so that it is possible to solve for $T_{c}$. It is a self-consistent condition that the number of pairs that $\mathrm{BE}$ condense is $\propto T_{c}$ itself. This may be why we can obtain the transition temperature without prior knowledge of the interaction mechanism. This works best for short coherence length pairs, and not so well for metallics with the greatest pair-pair interactions because of their long coherence lengths with a large overlap of pairs.

${ }^{1}$ D. S. Betts, Contemp. Phys. 10, 241 (1969).

${ }^{2}$ K. Huang, Statistical Mechanics (Wiley, New York, 1963).

${ }^{3}$ J. M. Blatt, Theory of Superconductivity (Academic, New York, 1964).

${ }^{4}$ P. C. Hohenberg, Phys. Rev. 158, 383 (1967).

${ }^{5} \mathrm{M}$. Rabinowitz, in Proceedings of the EPRI Workshop on HighTemperature Superconductivity, 1987, pp. 2-3 to 2-33, EL/ER-5894.

${ }^{6} \mathrm{M}$. Rabinowitz, in Proceedings of the EPRI Conference on Electrical Applications of Superconductivity; 1988, pp. 1-1 to 1-18, EL-6325-D.

${ }^{7}$ M. Rabinowitz, Int. J. Theor. Phys. 28, 137 (1989).

${ }^{8}$ M. Rabinowitz, Physica C 162-164, 249 (1989).

${ }^{9}$ M. Rabinowitz, Advances in Cryogenic Engineering (Plenum, New York, 1990), Vol. 36A, p. 21.

${ }^{10}$ M. Rabinowitz, Int. J. Theor. Phys. 32, 565 (1993).

${ }^{11}$ M. Rabinowitz, Appl. Phys. Commun. 12, 55 (1993).

${ }^{12}$ D. R. Harshman and A. P. Mills, Phys. Rev. B 45, 10684 (1992).

${ }^{13}$ M. R. Schafroth, Phys. Rev. 100, 463 (1955).

${ }^{14}$ M. R. Schafroth, S. T. Butler, and J. M. Blatt, Helv. Phys. Acta 30, 93 (1957). 